\title{
Modified Protocol of Harvesting, Extraction, and Normalization Approaches for Gas Chromatography Mass Spectrometry-Based Metabolomics Analysis of Adherent Cells Grown Under High Fetal Calf Serum Conditions
}

\author{
Raphaela Fritsche-Guenther ${ }^{1,2}$, Anna Bauer ${ }^{1,2}$, Yoann Gloaguen 1,2,3®0, Mario Lorenz ${ }^{4,5}(\mathbb{D}$ and \\ Jennifer A. Kirwan $1,2, *$ (1) \\ 1 Berlin Institute of Health Metabolomics Platform, Berlin Institute of Health (BIH), 10178 Berlin, Germany; \\ raphaela.fritsche@mdc-berlin.de (R.F.-G.); anna.bauer@mdc-berlin.de (A.B.); \\ yoann.gloaguen@mdc-berlin.de (Y.G.) \\ 2 Max-Delbrück-Center for Molecular Medicine (MDC) in the Helmholtz Association, 13125 Berlin, Germany \\ 3 Core Unit Bioinformatics, Berlin Institute of Health (BIH), 10178 Berlin, Germany \\ 4 German Centre for Cardiovascular Research (DZHK), partner site Berlin, 13353 Berlin, Germany; \\ mario.lorenz@charite.de \\ 5 Charité-Universitätsmedizin Berlin, corporate member of Freie Universität Berlin, Humboldt-Universität zu \\ Berlin, and Berlin Institute of Health, Medizinische Klinik für Kardiologie und Angiologie, Campus Mitte, \\ 10117 Berlin, Germany \\ * Correspondence: Jennifer.kirwan@mdc-berlin.de; Tel.: +49- (0)30-9460-3088
}

Received: 13 November 2019; Accepted: 11 December 2019; Published: 18 December 2019

\begin{abstract}
A gas chromatography mass spectrometry (GC-MS) metabolomics protocol was modified for quenching, harvesting, and extraction of metabolites from adherent cells grown under high $(20 \%)$ fetal calf serum conditions. The reproducibility of using either $50 \%$ or $80 \%$ methanol for quenching of cells was compared for sample harvest. To investigate the efficiency and reproducibility of intracellular metabolite extraction, different volumes and ratios of chloroform were tested. Additionally, we compared the use of total protein amount versus cell mass as normalization parameters. We demonstrate that the method involving $50 \%$ methanol as quenching buffer followed by an extraction step using an equal ratio of methanol:chloroform:water (1:1:1, v/v/v) followed by the collection of $6 \mathrm{~mL}$ polar phase for GC-MS measurement was superior to the other methods tested. Especially for large sample sets, its comparative ease of measurement leads us to recommend normalization to protein amount for the investigation of intracellular metabolites of adherent human cells grown under high (or standard) fetal calf serum conditions. To avoid bias, care should be taken beforehand to ensure that the ratio of total protein to cell number are consistent among the groups tested. For this reason, it may not be suitable where culture conditions or cell types have very different protein outputs (e.g., hypoxia vs. normoxia). The full modified protocol is available in the Supplementary Materials.
\end{abstract}

Keywords: GC-MS; 20\% FCS; harvesting; extraction; metabolites; normalization

\section{Introduction}

Reproducibility and robustness when performing metabolomics experiments are essential to guarantee that resulting biological information is both accurate and meaningful, and to reduce the likelihood of false discovery due to technical variation [1]. Cell cultures are often used as models for testing biological concepts. Therefore, an optimized and standardized protocol for efficient 
quenching, harvesting, and extraction of cells is required. There are many studies focusing on sample preparation methods, and many cell-dependent methods have been optimized for the application of cell metabolomics for analyzing metabolic fingerprints [2-10]. However, no preparation method is broadly applicable for all cell types or cultivation conditions. We had previously discovered that, in our hands, certain cell extraction methods were inappropriate for the cell types we were using, typically because they did not adequately separate the protein layer from the solvents. This was assumed to be a result of inappropriate volumes and ratios of the solvents to adequately extract the cell lipid content.

To prepare cells for metabolomics analyses, quenching at the point of harvesting aims to inactivate intracellular enzymes and stop metabolism to avoid degradation and alterations of sample composition. Sample preparation should be highly reproducible, robust, and fast to allow high-throughput studies [5]. As is known, metabolic reactions occur in milliseconds [11]. Therefore, it is necessary to quench metabolism quickly, most often by using ice-cold organic solvents. After the quenching and harvesting step, metabolite extraction is crucial and often the rate-limiting step. Extraction is the process by which specific compounds, or whole classes of compounds, e.g., polar metabolites are selectively separated from others (e.g., lipids, proteins) and is normally conducted to yield cleaner sample preparations for analysis. There has been much discussions over which extraction solvents are the best for quenching and measurement of metabolites [12]. Biphasic, liquid-liquid extraction is often used to extract metabolites, typically based on Folch or Bligh-Dyer methods [13-15]. Polar solutions like methanol $(\mathrm{MeOH})$ in water $\left(\mathrm{H}_{2} \mathrm{O}\right)$ are often paired with nonpolar organic solvents, such as chloroform $\left(\mathrm{CHCl}_{3}\right)$, to form a two-phase system. The chemical properties, volumes, and solvent ratios of the organic and aqueous solvents must be considered carefully. These parameters can significantly affect the extraction efficiency of metabolites and the experimental reproducibility.

Fetal calf serum (FCS) contains a large number of nutritional and macromolecular factors such as amino acids, sugars, lipids, and hormones essential for cell culture growth. For most cell lines, 10\% FCS is used; however, some primary cells, such as human umbilical vein endothelial cells (HUVECs), need to be cultured in higher FCS conditions (e.g., medium with 20\% FCS) [16-22]. To our knowledge, there have been no reports to date documenting the robustness and efficiency of harvesting and extraction protocols for metabolic profiling of adherent cells grown under high FCS conditions. Ideally, this would be undertaken using a full design-of-experiments approach investigating and fully testing multiple parameters to optimize the method. However, due to limitations in both resources and numbers of primary cell cultures, an incremental optimization approach can be taken to reach a predefined reproducibility and metabolite detection threshold. This approach enables an early exclusion of conditions that do not fit one's criteria, therefore making the most out of the material available.

Therefore, the aim of this study was to compare and enhance our existing protocol for a reliable and reproducible harvesting and extraction of adherent cells grown in media supplemented with $20 \%$ FCS. As a comparison, standard cell culture conditions (10\% FCS) were also investigated. All analyses were conducted by gas chromatography-mass spectrometry (GC-MS). The use of either 50\% or $80 \% \mathrm{MeOH}$ for quenching of cells were compared for sample harvest. In addition, two different ratios and three different final volumes of $\mathrm{MeOH}$ to $\mathrm{CHCl}_{3}$ to $\mathrm{H}_{2} \mathrm{O}$ were compared to investigate the efficiency and reproducibility of intracellular metabolite extraction. To achieve robust normalization of the samples, total protein amount versus absolute cell mass was tested.

\section{Results}

\subsection{Preliminary Experiments on Phase Separation}

A previous protocol used in our lab for adherent cells growing under 10\% FCS was a modified Bligh-Dyer extraction to separate lipid and polar metabolites [23,24]. The protocol includes the use of $50 \% \mathrm{MeOH}$ for cell quenching followed by an extraction using a 1:0.4:1, v/v/v MeOH:CHCl $3: \mathrm{H}_{2} \mathrm{O}$ ratio. Routinely, $3 \mathrm{~mL}$ of the polar phase is collected and used for further GC-MS measurement. For HUVECs cultured under conditions of $20 \%$ FCS, we discovered that this protocol was not sufficient 
to properly extract the cells. We discovered a fluffy protein pellet rather than the compacted, well demarcated protein pellet we normally achieve. This had consequences for the reproducibility of the measured total protein amount resulting in a relative standard deviation (RSD) for HUVECs of over 25\% (European Medicine Agency (EMA) recommendation is RSD < 15\%, http://www.ema.europa.eu/). To check this was not a cell-specific effect, the HCT116 colorectal carcinoma cell line was cultured and extracted in an identical way. Results were similar for the HCT116 cells with total protein RSDs also over 25\% for high and standard FCS conditions, as seen in Table 1 and Figure 150_LOW. This suggests that extraction may not be complete and thus the technical reproducibility was larger than necessary. This prompted us to look at the entire process of harvesting and extraction.

In our study, four different extraction conditions were tested. The four conditions can be summarized here as: (1) 50_LOW: $50 \% \mathrm{MeOH}$ quenching solution with $1 \mathrm{~mL}$ of $\mathrm{CHCl}_{3}$ for extraction with a final ratio of 1:0.4:1 ( $\left.\mathrm{MeOH}: \mathrm{CHCl}_{3}: \mathrm{H}_{2} \mathrm{O}\right)$ and collection of $3 \mathrm{~mL}$ polar phase for analysis; (2) 50_MEDIUM: 50\% MeOH quenching solution with $2.5 \mathrm{~mL}$ of $\mathrm{CHCl}_{3}$ for extraction with a final ratio of 1:1:1 (MeOH: $\mathrm{CHCl}_{3}: \mathrm{H}_{2} \mathrm{O}$ ) and collection of $3 \mathrm{~mL}$ polar phase for analysis; (3) 80_HIGH: $80 \% \mathrm{MeOH}$ quenching solution with $4 \mathrm{~mL}$ of $\mathrm{CHCl}_{3}$ for extraction with a final ratio of 1:1:1 $\left(\mathrm{MeOH}: \mathrm{CHCl}_{3}: \mathrm{H}_{2} \mathrm{O}\right)$ and collection of $6 \mathrm{~mL}$ polar phase for analysis; and (4) 50_HIGH: 50\% MeOH quenching solution with $4 \mathrm{~mL}$ of $\mathrm{CHCl}_{3}$ for extraction with a final ratio of 1:1:1 (MeOH:CHCl $\left.3: \mathrm{H}_{2} \mathrm{O}\right)$ and collection of $6 \mathrm{~mL}$ polar phase for analysis.

HCT116

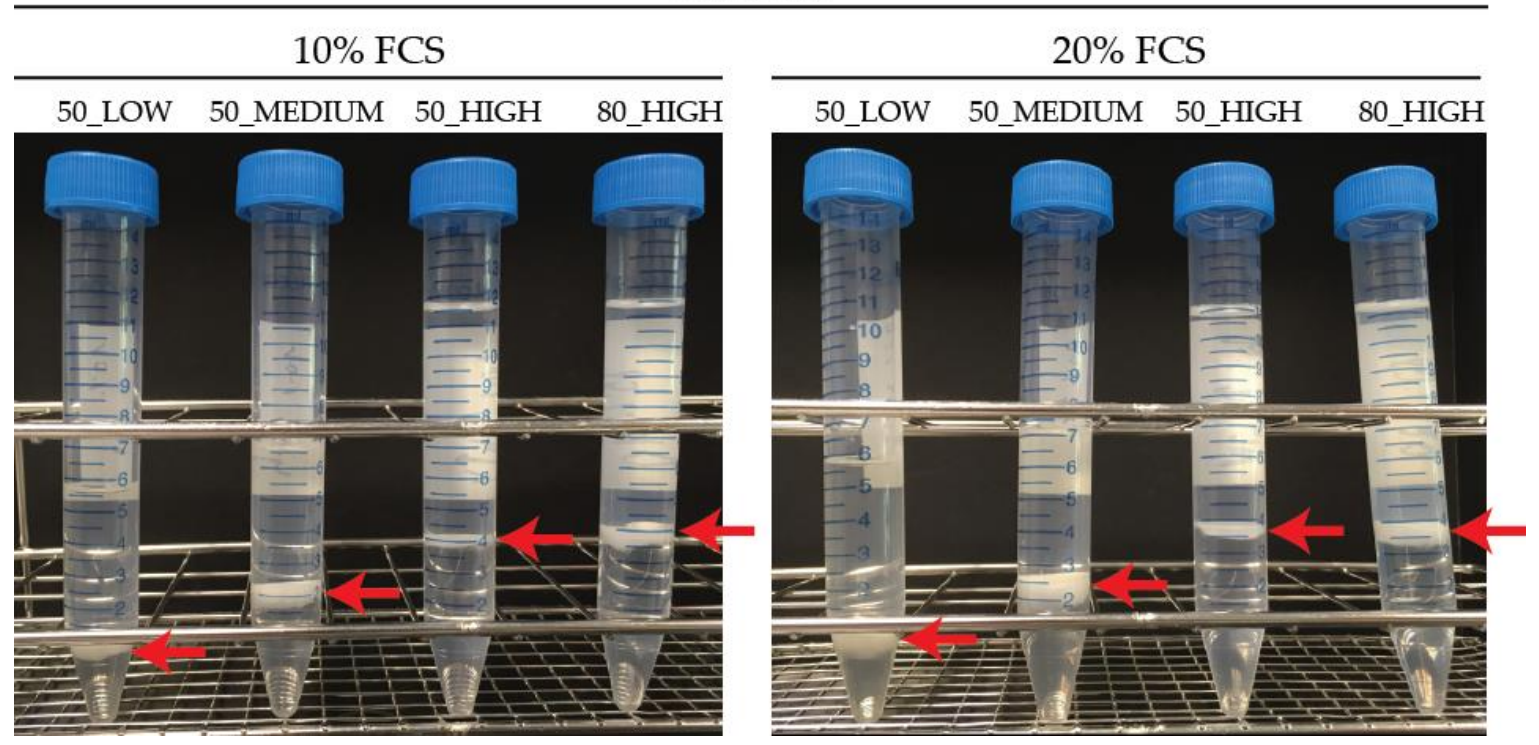

Figure 1. Extraction of HCT116 cells cultured in $10 \%$ and $20 \%$ fetal calf serum (FCS) conditions using different quenching solvents, extraction ratios, and polar volumes (protein layer shown by extra arrow on picture).

Table 1. Relative standard deviation (RSD) of measured protein amount of HCT116 cells cultured in $10 \%$ and $20 \%$ FCS conditions using different quenching solvents, extraction ratios, and polar volumes.

\begin{tabular}{ccccc}
\hline Condition Name & 50_LOW & 50_MEDIUM & 50_HIGH & 80_HIGH \\
\hline Biological replicates 10\% FCS & 4 & 3 & 4 & 5 \\
Biological replicates 20\% FCS & 5 & 3 & 4 & 4 \\
\hline HCT116 (10\% FCS) & 51 & 20 & 14 & 13 \\
\hline HCT116 (20\% FCS) & 30 & 42 & 16 & 8 \\
\hline
\end{tabular}




\subsection{Experiment 1: Determining the Optimum Concentration of $\mathrm{MeOH}$ as a Quenching and Extraction Solution for Cell Harvesting}

The quenching solution used is important as it has a dual function of being the first extraction step and being required to quench metabolism quickly. Many reports suggest $80 \% \mathrm{MeOH}$ for metabolite quenching and extraction [7,25,26]; our current protocol uses 50\% MeOH (50_LOW). Therefore, both $50 \%$ and $80 \% \mathrm{MeOH}$ solutions were tested as quenching and extraction buffers for harvesting of adherent cells cultured in 10\% and $20 \%$ FCS. The modified Bligh-Dyer extraction step which follows the quenching step will influence the results. In a first test, we used the same volume of $1 \mathrm{~mL} \mathrm{CHCl}_{3}$ for the extraction step (as used in our current protocol) and observed that for $80 \% \mathrm{MeOH}$, no phase separation occurred, as seen in Figure S1. This is assumed to be due to the relative ratios of $\mathrm{CHCl}_{3}$ and to polar solvents. Increasing the ratio of $\mathrm{MeOH}: \mathrm{CHCl}_{3}: \mathrm{H}_{2} \mathrm{O}$ to 1:1:1 $/ / v / v$ resulted in a phase separation. In order to keep the ratio of the final solvent composition constant, the extracted volumes of $\mathrm{H}_{2} \mathrm{O}$ and $\mathrm{MeOH}$ needed to be adjusted accordingly to account for whether $50 \%$ or $80 \% \mathrm{MeOH}$ was used in the first extraction step.

The performance of individual quenching solvents was determined by a range of measures including (i) number of detected metabolites, (ii) median RSD of all detected metabolites (a measure of reproducibility), (iii) the percentage of metabolites with an individual RSD $<30 \%$ (a generally accepted tolerance limit for GC-MS metabolomics for any individual metabolite [27]), and (iv) the sum of normalized peak area for all annotated metabolites (henceforth referred to as sum of area), a proxy measure of the overall sensitivity of the method.

In the HCT116 cells, the conditions were equivalent in terms of number of metabolites detected, as seen in Figure S2, except for the detection of fructose-6-phosphate, when $80 \% \mathrm{MeOH}$ was used with 10\% culture conditions, as seen in Table S1, comparing 50_HIGH and 80_HIGH. For the HUVECs, a lower number of metabolites was found, as seen in Table S2 and Figure S2; 34 metabolites for 50_HIGH and 36 for 80_HIGH. We assume this lower number of metabolites is due to the generally lower average cell count achieved by the HUVECs (mean cell counts: male HUVECs: $1.5 \times 10^{6}$ cells; female HUVECs: $3 \times 10^{6}$ cells; HCT116: $6 \times 10^{6}$ cells). The metabolites detected also had some very minor differences (e.g., proline was only detected in 50_HIGH and tyrosine, uracil, and ribose-5-phosphate were only detected in 80_HIGH).

The reproducibility of both quenching solvents was then assessed using RSDs as an indicator. For HCT116 cells and HUVECs in both culture conditions, 50\% MeOH performed better overall as a quenching solvent compared to 80\% MeOH, comparing 50_HIGH and 80_HIGH in Table 2, Table 3, Figure 2, Table S1, Table S2, Table S3, Table S4, and Figure S3. In HCT116 cells 50_HIGH has a lower median RSD compared to 80_HIGH (14\% compared to 23\%, and 26\% compared to 30\%, for $10 \%$ FCS and $20 \%$ FCS, respectively). In addition, there was a higher percentage of individual metabolites with RSD $<30 \%$ in the 50_HIGH (79\% compared to $67 \%$, and $73 \%$ compared to $53 \%$, for $10 \%$ FCS and $20 \%$ FCS, respectively). In line, the sum of the area was higher in 50_HIGH compared to 80_HIGH for both FCS culture conditions (Figure 3; 74 compared to 56, and 35 compared to 26, for 10\% FCS and 20\% FCS, respectively). In HUVECs, the median RSD was lower in 50_HIGH (13\%) compared to 80_HIGH conditions $(36 \%)$, with a higher percentage of individual metabolite with RSDs < 30\% (74\% 50_HIGH compared to 44\% 80_HIGH). The measured sum of area for HUVECs was also better for 50_HIGH (3.8 compared to 3.2) According to these results, $50 \% \mathrm{MeOH}$ was deemed to be a better quenching solvent for both types of cells. However, for particular metabolites, 80\% $\mathrm{MeOH}$ may be more appropriate. 
Table 2. Median relative standard deviation (RSD) per metabolite of HCT116 cells cultured in $10 \%$ and $20 \%$ FCS conditions using different quenching solvents, extraction ratios, and polar volumes.

\begin{tabular}{ccccc}
\hline Condition Name & 50_LOW & 50_MEDIUM & 50_HIGH & 80_HIGH \\
\hline Biological replicates 10\% FCS & 4 & 3 & 4 & 5 \\
Biological replicates 20\% FCS & 5 & 3 & 4 & 4 \\
\hline HCT116 (10\% FCS) & $68 \%$ & $22 \%$ & $14 \%$ & $23 \%$ \\
\hline HCT116 (20\% FCS) & $33 \%$ & $55 \%$ & $26 \%$ & $30 \%$ \\
\hline
\end{tabular}

Table 3. Percentage of metabolites with a relative standard deviation (RSD) $<30 \%$ of HCT116 cells cultured in $10 \%$ and $20 \%$ FCS conditions using different quenching solvents, extraction ratios, and polar volumes.

\begin{tabular}{ccccc}
\hline Condition Name & 50_LOW & 50_MEDIUM & 50_HIGH & 80_HIGH \\
\hline Biological replicates 10\% FCS & 4 & 3 & 4 & 5 \\
Biological replicates 20\% FCS & 5 & 3 & 4 & 4 \\
\hline HCT116 (10\% FCS) & $0 \%$ & $73 \%$ & $79 \%$ & $67 \%$ \\
\hline HCT116 (20\% FCS) & $15 \%$ & $3 \%$ & $73 \%$ & $53 \%$ \\
\hline
\end{tabular}

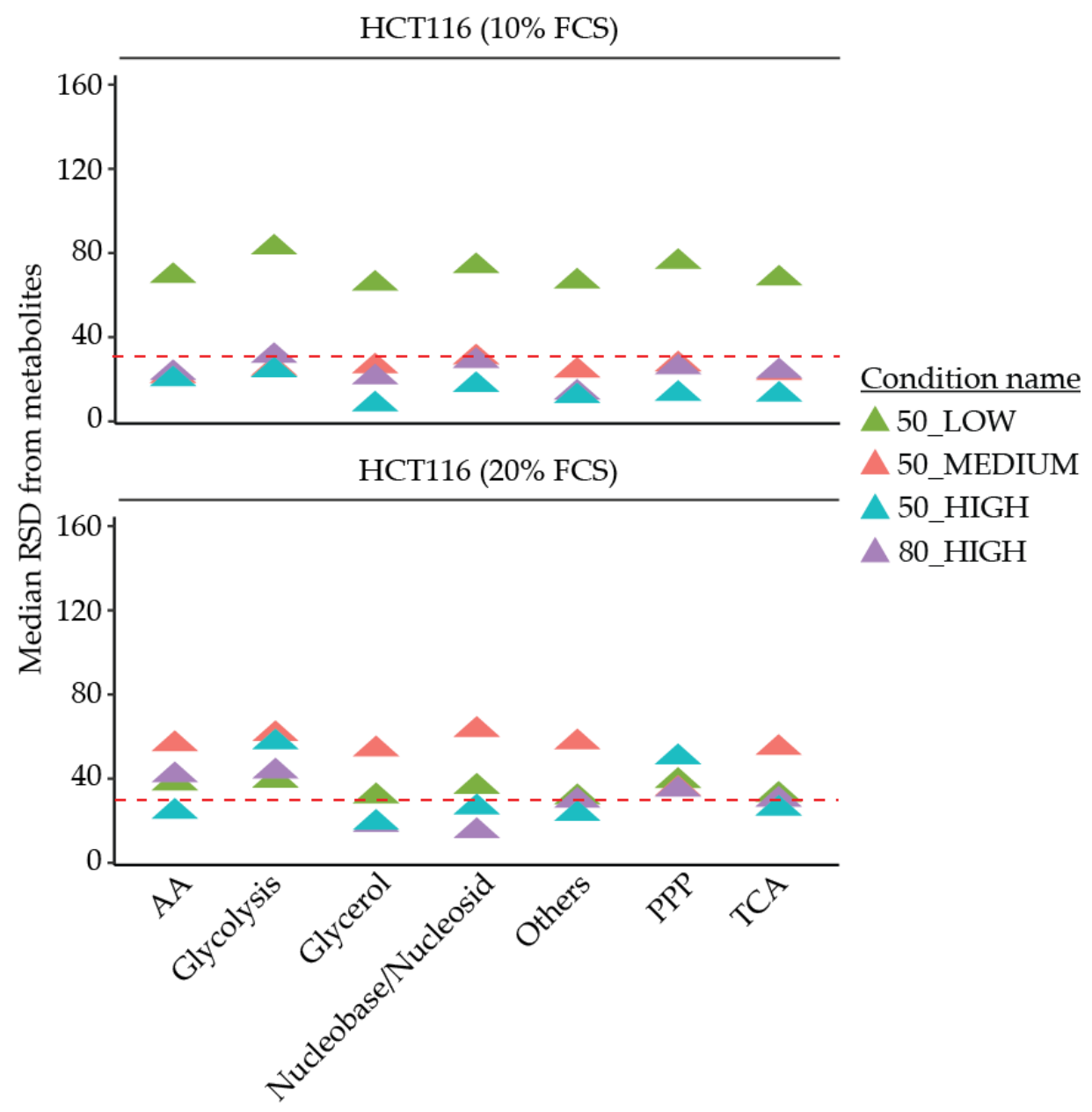

Figure 2. Median relative standard deviation (RSD) of individual metabolites separated by biological metabolite pathways of HCT116 cells cultured in 10\% and 20\% FCS conditions using different quenching solvents, extraction ratios, and polar volumes. The dashed line represents the maximum $30 \%$ RSD threshold advised by the Federal Drug Administration (FDA). AA: Amino acids. PPP: Pentose phosphate pathway. TCA: Tricarboxylic acid cycle. 
a

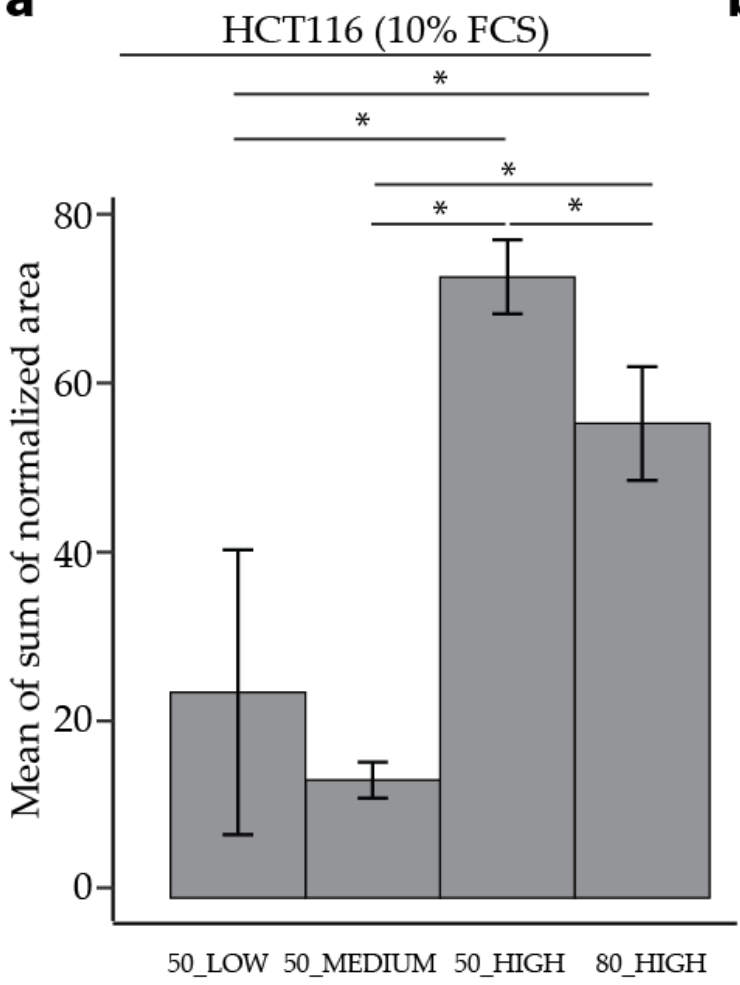

b

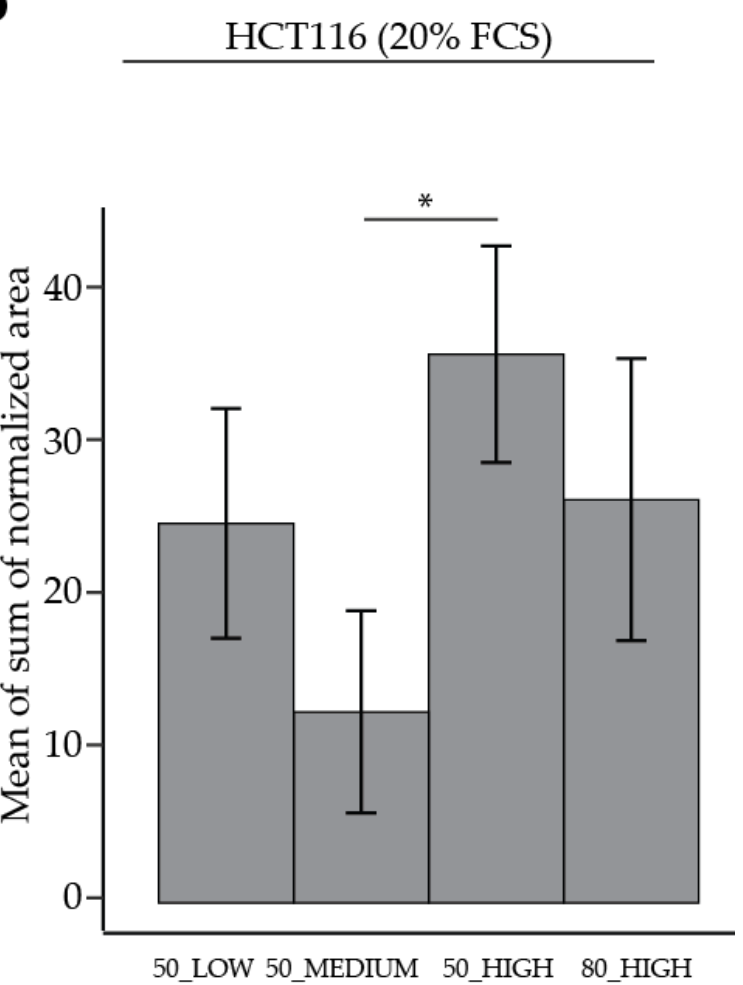

Figure 3. Comparison of the mean of sum of normalized peak area for different quenching and extraction methods. Data from a minimum three out of five HCT116 cells cultured in (a) $10 \%$ and (b) 20\% FCS. The error bars represent the standard deviation of the biological replicates. Samples were analyzed using an unpaired Student's $t$-test with $p<0.05$ deemed as statistically significant.

2.3. Experiment 2: Determination of the Optimal Volume and Ratio of $\mathrm{MeOH}: \mathrm{CHCl}_{3}: \mathrm{H}_{2} \mathrm{O}$ for a Metabolite Extraction Solution

The ratio of $\mathrm{MeOH}: \mathrm{CHCl}_{3}: \mathrm{H}_{2} \mathrm{O}$ is important for: (i) the efficiency and reproducibility of extraction; (ii) a clear phase separation; and (iii) a robust method for protein collection for normalization or for concurrent proteomics (see Section 2.1). Different ratios, final volumes, and polar phase volume collection for extraction were evaluated, as seen in Table 4. This step was carried out before 50_HIGH was deemed to be optimum for quenching, but, for convenience, is presented here in the same order in which it appears in the standard operating protocol. As the different extraction methods led to different final volumes, leading to different theoretical concentrations, more volume was analyzed in some samples to normalize and compensate for this concentration difference.

Table 4. Experimental set up for the conditions analyzed in the study.

\begin{tabular}{|c|c|c|c|c|}
\hline Condition Name & 50_LOW (A) & 50_MEDIUM (A) & 50_HIGH (A) & 80_HIGH (A) \\
\hline $5 \mathrm{~mL}$ of $\mathrm{MeOH}$ harvest & $\begin{array}{c}50 \% \\
(2.5+2.5) *\end{array}$ & $\begin{array}{c}50 \% \\
(2.5+2.5) *\end{array}$ & $\begin{array}{c}50 \% \\
(2.5+2.5) *\end{array}$ & $\begin{array}{c}80 \% \\
(4.0+1.0) *\end{array}$ \\
\hline $\mathrm{MeOH}[\mathrm{mL}]$ for extraction & - & - & 1.5 & - \\
\hline $\mathrm{H}_{2} \mathrm{O}[\mathrm{mL}]$ for extraction & - & - & 1.5 & 3.0 \\
\hline $\mathrm{CHCl}_{3}[\mathrm{~mL}]$ for extraction & 1.0 & 2.5 & 4.0 & 4.0 \\
\hline Final volume $[\mathrm{mL}]$ & 6.0 & 7.5 & 12 & 12 \\
\hline Final ratio $v / v / v$ & $1: 0.4: 1$ & $1: 1: 1$ & $1: 1: 1$ & $1: 1: 1$ \\
\hline Final polar volume $[\mathrm{mL}]$ & 5.0 & 5.0 & 8.0 & 8.0 \\
\hline Used polar volume [mL] & 3.0 & 3.0 & 6.0 & 6.0 \\
\hline
\end{tabular}


The evaluation of the different ratios and volumes revealed no significant changes in either the number of detected metabolites, as seen in Table 5, or particular metabolite classes independent of the analyzed cells and growth conditions, as seen in Figure S2.

Table 5. Number of annotated central carbon metabolites of HCT116 cells cultured in $10 \%$ and $20 \%$ FCS conditions using different quenching solvents, extraction ratios, and polar volumes.

\begin{tabular}{ccccc}
\hline Condition Name & 50_LOW & 50_MEDIUM & 50_HIGH & 80_HIGH \\
\hline Biological replicates 10\% FCS & 4 & 3 & 4 & 5 \\
Biological replicates 20\% FCS & 5 & 3 & 4 & 4 \\
\hline HCT116 (10\% FCS) & $38 / 45$ & $39 / 45$ & $38 / 45$ & $39 / 45$ \\
\hline HCT116 (20\% FCS) & $41 / 45$ & $41 / 45$ & $40 / 45$ & $40 / 45$ \\
\hline
\end{tabular}

In HCT116 cells, the use of 50_MEDIUM compared to 50_LOW led to lower RSDs (20\% compared to $51 \%$ ) for total protein measurement in cells grown under 10\% FCS. HCT116 cells cultured with $20 \%$ FCS showed increased RSDs (42\% compared to 30\%) in the 50_MEDIUM condition, as seen in Table 1. When using 80_HIGH conditions, the pellet was more compact, as seen in Figure 1, and easier to extract, which is reflected in a low RSD (13\% for $10 \%$ FCS and $8 \%$ for $20 \%$ FCS) of the measured protein amount in HCT116 cells. When using 50_HIGH conditions, a compact protein pellet could be observed, leading to a more efficient protein extraction, and resulting in RSDs (14\% for 10\% FCS and $16 \%$ for $20 \%$ FCS) similar to $80 \_$HIGH conditions. For HUVECs, the RSD of the measured total protein was reduced when using 80_HIGH compared to 50_MEDIUM and 50_HIGH conditions, as seen in Table S5. 50_MEDIUM compared to 50_LOW conditions showed a lower RSD (15\% compared to $49 \%$, respectively).

The reproducibility of GC-MS analysis as measured by the median RSD of the individual metabolites or combined as biological pathways, and the percentage of metabolites with RSD $<30 \%$, revealed the best results when using 50_HIGH conditions for HCT116 cells, as seen in Table 2, Table 3 and Figure 2. Likewise, in HUVECs, the best results were shown using 50_HIGH conditions, as seen in Table S3, Table S4 and Figure S3.

Independent of the FCS setting in HCT116 cells, the highest sum of area was found using 50_HIGH conditions, as seen in Figure 3. For HUVECs, the highest sum of area was found in cells in the 50_MEDIUM condition; however, the variability was high, as seen in Figure S4. Comparing 80_HIGH and 50_HIGH conditions measured as a separate batch, a higher sum of area was found using 50\% $\mathrm{MeOH}$ as quenching buffer.

In summary, our results indicate that using an extraction ratio of $1: 1: 1, v / v / v \mathrm{MeOH}: \mathrm{CHCl}_{3}: \mathrm{H}_{2} \mathrm{O}$ and a polar phase volume of $6 \mathrm{~mL}$ achieved good results for samples used for both GC-MS measurement and protein extraction for cells grown under 10\% and 20\% FCS. Lower individual and combined RSDs and higher peak areas could be detected. The direct comparison of $50 \%$ or $80 \% \mathrm{MeOH}$ as quenching buffer showed a higher sum of area and lower median RSDs when using 50_HIGH conditions for the analyzed cells and FCS conditions. The number of annotated metabolites was equal across all methods. The full modified protocol is available in the Supplementary Materials.

\subsection{Experiment 3: Total Protein versus Cell Mass as a Normalization Strategy}

Adherent cells need to be detached from the bottom of the cell culture flask for further metabolomics analyses. Trypsinization is known to lead to alteration of metabolism, so we employed direct quenching of cells with physical scraping as an alternative $[5,28]$. However, this complicates the utilization of the cell count as a normalization parameter. Therefore, we compared the use of total protein amount versus the cell mass as a normalization strategy.

After normalizing to total protein, HCT116 cells grown under 10\% FCS showed a slightly lower median RSD of $24 \%$ for individual metabolite detection compared to normalization with cell mass 
(median RSD 28\%), as seen in Table 6. For HCT116 cells cultivated in 20\% FCS, a median RSD of 35\% and $25 \%$, respectively, was found if normalized to total protein or cell mass. For HUVECs, the median RSD was lower when using protein (52\%) for normalization compared to cell mass $(61 \%)$, as seen in Table S6. The percentage of individual metabolite RSDs $<30 \%$ threshold was higher when using total protein (26\%) compared to cell mass (18\%) for normalization. Judging from Table 6 and Table S6, neither of the normalization methods was superior. However, total protein was considerably easier to measure.

Table 6. Median relative standard deviation (RSD) and percentage of metabolites with a RSD $<30 \%$ from four replicates of HCT116 cells cultured in $10 \%$ and $20 \%$ FCS conditions.

\begin{tabular}{ccccc}
\hline & \multicolumn{2}{c}{ HCT116 (10\% FCS) } & \multicolumn{2}{c}{ HCT116 (20\% FCS) } \\
& Cell Mass & Protein & Cell Mass & Protein \\
\hline Median RSD $[\%]$ & 28 & 24 & 25 & 35 \\
\hline RSD $<30 \%[\%]$ & 55 & 60 & 60 & 33 \\
\hline
\end{tabular}

\section{Discussion}

Metabolomics has long been used for the analysis of human body fluids for clinical indications [29,30]. More recently, it is also being applied to cells or tissue [30]. Metabolite profiling of adherent growing mammalian cells is challenging, particularly due to the special requirements with respect to the sampling procedure. While harvesting of suspension cultures can generally be achieved through rapid centrifugation, the adherent cells first need to be detached from the bottom of the cell culture flask [30]. With a large number of metabolites to be analyzed simultaneously, it becomes difficult to find a single optimal extraction method. In this work, we set out to solve a particular challenge, which was the visible suboptimal extraction of cells grown under high (20\%) FCS conditions. We focused on optimizing a liquid-liquid extraction protocol for the harvest and extraction of cells grown both under high $(20 \%)$ or normal $(10 \%)$ FCS conditions to enable reproducible and robust analysis of the detected metabolites. We compared our existing protocol using $50 \% \mathrm{MeOH}$ to $80 \% \mathrm{MeOH}$ as a quenching buffer, followed by an extraction using different ratios and volumes of $\mathrm{CHCl}_{3}$ and polar phases. Environmental and personal safety should also be considered and has resulted in a move away from $\mathrm{CHCl}_{3}$ towards other nonorganic solvents such as methyl-tert-butylether (MTBE) in recent years [31]. MTBE is both highly volatile and floats on the surface of the resulting biphasic mixture. As we were interested in analyzing only the polar metabolites in this study, it was excluded from the test solvents. We also looked at whether cell mass or total protein represented a better normalization strategy, since cell count cannot easily be used for adherent cells harvested for a metabolomics approach [32].

Direct quenching of the metabolism in adherent cells needs extraction with organic solvents such as $\mathrm{MeOH}$. Various reports on the best solvent composition for metabolite extraction suggests $80 \% \mathrm{MeOH}$ in $\mathrm{H}_{2} \mathrm{O}[7,26]$; however, $50 \% \mathrm{MeOH}$ is also used for metabolite extraction of adherent cells grown under 10\% FCS [23]. We tried using both $50 \% \mathrm{MeOH}$ and $80 \% \mathrm{MeOH}$ as quenching buffers, followed by an extraction using different ratios and volumes of $\mathrm{CHCl}_{3}$ and polar phases in cells cultured with $20 \%$ FCS compared to standard cell culture conditions. Using the 50_HIGH method (50\% MeOH followed by extraction using a 1:1:1, v/v/v MeOH:CHCl $3: \mathrm{H}_{2} \mathrm{O}$ ratio and collection of $6 \mathrm{~mL}$ polar phase) was considered to be the best of the extraction methods we tested. Using a higher ratio of $\mathrm{CHCl}_{3}$ and higher extraction solvent volumes gives a better phase separation and results in a dense protein pellet allowing a robust normalization. The percentage of highly polar solutions in the final ratio is also likely to be important for efficient extraction of polar metabolites and is reflected in the quenching results. The results may be different if nonpolar metabolites are also considered.

Normalization methods for cell culture are often problematic. Normalization is used to eliminate inter-run variability and biological variation [25]. Cell counts are inaccurate and are often performed on separate culture plates to the ones that are analyzed [33]. This is labor-intensive, impractical, and 
inefficient, especially with regard to large studies. Using cell count for normalization requires cell detachment using trypsin solutions, which can lead to metabolite leakage and changes in the metabolic pattern [5]. Total protein or cell mass has the advantage over cell counts that they can be performed using the same plate as that for the metabolomics analysis. One difficulty in using cell mass for normalization is the removal of the quenching buffer after centrifugation. Remnants of the quenching solution left in the vials will affect the final recorded cell mass, leading to more technical error. Further, the process of weighing the samples is technically laborious. Metabolites are contained in the buffer, meaning that it needs to be first removed for the weighing process and then re-added. This all needs to take place as quickly as possible, while keeping the sample as cold as possible so that it has minimal effects on reproducibility. As total protein can be measured following extraction, it can be done with more ease, although it has the disadvantage that samples cannot then be extracted according to their mass. They can, however, be reconstituted to equivalent concentrations before being analyzed if a drying down step is employed immediately after extraction. We have shown in the results that the extraction of the protein was reproducible enough to be considered an effective normalization method when the 50_HIGH method was used. For large numbers of samples, protein extraction is easier since the critical step of weighing and cooling is missing. Therefore, we recommend using total protein as a potential normalization strategy. We are aware of certain cell culture conditions that reduce the protein amount per cell. For this reason, full consideration should be given to whether the total protein is likely to be a good reflection of cell count or cell volume before it is used. In our hands, we have seen certain culture conditions, e.g., hypoxia, where cell protein production is not equivalent in the same cell type as in normoxia. In these circumstances, cell protein would probably not be recommended as a suitable normalization strategy.

In this study, we have tested and improved our existing method to enhance our reproducibility when analyzing adherent mammalian cells cultured under $20 \%$ FCS. For a truly optimized method, a design of experiments (DoE) approach is recommended. However, due to the difficulties of working with large numbers of cell cultures per batch, the cost of repeated experiments and the statistical understanding required to pursue a DoE approach, we instead chose to trial and optimize our existing method. We are aware that this incremental optimization approach may not result in the truly optimal method; however, it enables a faster development that limits material waste. The method is deemed to be optimal once certain reproducibility and metabolite detection criteria are reached. This represents a more realistic scenario for the thousands of labs required to run samples each year without the time and resources to fully optimize methods for each sample type.

In summary, the final modified 50_HIGH quenching, extraction, and normalization method (50\% $\mathrm{MeOH}$ for harvest followed by extraction using a 1:1:1, v/v/v MeOH:CHCl$: \mathrm{H}_{2} \mathrm{O}$ ratio and collection of $6 \mathrm{~mL}$ polar phase) can be recommended for the investigation of intracellular metabolites from adherent human cells grown under standard (10\%) or high (20\%) FCS conditions using a GC-MS platform.

\section{Materials and Methods}

\subsection{Cell Culture}

Two different human cell types were used in this study. We were interested mainly in a robust analysis of HUVEC male and female twin pair cells, but since these are human derived and therefore in limited supply, HCT116 cells were used to enable more replicates to be tested. For the same reason, a mix of male and female HUVECs were used.

The colorectal carcinoma cell line HCT116 was obtained from ATCC (American Type Culture Collection, Teddington, UK). The HCT116 cell line was maintained in DMEM (Dulbecco's Modified Eagle Medium, Thermo Fischer Scientific, Waltham, MA, USA) supplemented with 10\% or $20 \%$ FCS (Thermo Fischer Scientific, Waltham, MA, USA), 1\% penicillin/streptomycin (Thermo Fischer Scientific, Waltham, MA, USA), 2 mM glutamine (Thermo Fischer Scientific, Waltham, MA, USA) and 1 g/L 
glucose (Sigma-Aldrich, St. Louis, MO, USA). HUVECs were isolated as previously described [17]. All cells were incubated in a humidified atmosphere of $5 \% \mathrm{CO}_{2}$ in air at $37^{\circ} \mathrm{C}$.

\subsection{Experiment 1: Determination of the Optimum Concentration of Methanol as a Quenching and Extraction} Solution for Cell Harvesting

Cells were rapidly washed ( $20 \mathrm{~s}$ ) with washing buffer ( $140 \mathrm{mM} \mathrm{NaCl}, 5 \mathrm{mM}$ HEPES, $\mathrm{pH} 7.4,37^{\circ} \mathrm{C}$ ) before they were quenched by ice-cold $\mathrm{MeOH}$ solution. Two solutions were tested: either $5 \mathrm{~mL}$ of $50 \%$ or $80 \% \mathrm{MeOH}$ in $\mathrm{H}_{2} \mathrm{O}$ with a final concentration of $2 \mu \mathrm{g} / \mathrm{mL}$ cinnamic acid (for use as an internal standard). Immediately as the $\mathrm{MeOH}$ solution was added to the culture plate, cells were scraped into the methanol solution and the methanolic lysates were collected. The extracts were agitated to complete cell lysis and centrifuged to separate the layers (see Section 4.3).

\subsection{Experiment 2: Determination of the Optimal Volume and Ratio of $\mathrm{MeOH}: \mathrm{CHCl}_{3}: \mathrm{H}_{2} \mathrm{O}$ for a Metabolite Extraction Solution}

After cell harvest, $\mathrm{CHCl}_{3}$ was added to the methanolic cell extracts, shaken for $60 \mathrm{~min}$ at $4{ }^{\circ} \mathrm{C}$, and centrifuged at $4149 \times \mathrm{g}$ for $10 \mathrm{~min}$ at $4{ }^{\circ} \mathrm{C}$ to separate the phases. Both the final ratio of $\mathrm{MeOH}: \mathrm{CHCl}_{3}: \mathrm{H}_{2} \mathrm{O}$ and the final total volume was tested. Test conditions are summarized in Table 4.

The polar phase was collected and dried overnight at $30^{\circ} \mathrm{C}$ at a speed of $1550 \times g$ at 0.1 mbar using a rotational vacuum concentrator (RVC 2-33 CDplus, Christ, Osterode am Harz, Germany). Samples were pooled after extraction and used as a quality control $(\mathrm{QC})$ sample to test the technical variability of the instrument. QC samples were prepared alongside the samples in the same way. To generate backup samples, the dried polar phases were resuspended and split into two aliquots.

To further test whether the results of changing the ratios of $\mathrm{MeOH}: \mathrm{CHCl}_{3}: \mathrm{H}_{2} \mathrm{O}$ are an effect of the ratio or of the total volume, we investigated changing the ratios while maintaining the volume. For the harvesting condition of $50 \% \mathrm{MeOH}$, a final volume of $6 \mathrm{~mL}$ was tested, resulting in $3 \mathrm{~mL}$ of polar phase that could be collected for analysis. For the harvesting condition using $80 \% \mathrm{MeOH}$, a final extraction volume of $12 \mathrm{~mL}$ was tested with $6 \mathrm{~mL}$ polar phase collected and dried for analysis. To perform a direct comparison of the same volume we used $5 \mathrm{~mL}$ of $50 \% \mathrm{MeOH}$ for cell quenching followed by increased extraction volumes $\left(4 \mathrm{~mL} \mathrm{CHCl}_{3}\right.$, additional $1.5 \mathrm{~mL} \mathrm{MeOH}$ and $1.5 \mathrm{~mL} \mathrm{H}_{2} \mathrm{O}$ ) leading to a 1:1:1, v/v/v ratio of $\mathrm{MeOH}: \mathrm{CHCl}_{3}: \mathrm{H}_{2} \mathrm{O}$. With the increased volumes, $6 \mathrm{~mL}$ of polar phase could be collected and used for GC-MS measurement. The 80_LOW condition ( $5 \mathrm{~mL} 80 \% \mathrm{MeOH}$ for quenching and $1 \mathrm{~mL}$ of $\mathrm{CHCl}_{3}$ for extraction) was discarded from the optimization tests since no phase separation could be achieved, as seen in Figure S1.

\subsection{Experiment 3: Total Protein versus Cell Mass as a Normalization Strategy}

To measure cell mass, at the point immediately following quenching and harvest of the cells, the quenched cells $(50 \% \mathrm{MeOH})$ were centrifuged for $10 \mathrm{~min}$ at $10,000 \times g$ at $4{ }^{\circ} \mathrm{C}$ and the supernatant was carefully removed and collected into a new $15 \mathrm{~mL}$ Falcon tube stored on ice. The cell pellet, also kept on ice, was immediately weighed as fast as possible and the collected supernatant was added back to it, ready for metabolite extraction. The metabolites were extracted using a high volume of $\mathrm{CHCl}_{3}$ $(4 \mathrm{~mL})$ as determined in the previous section. After collection of the polar phases $(6 \mathrm{~mL})$, proteins were precipitated for each sample by addition of $8 \mathrm{~mL} 100 \% \mathrm{MeOH}$ to avoid phase separation followed by centrifugation at $16,000 \times g$ speed for $10 \mathrm{~min}$. The supernatant was carefully discarded. The pellet was air dried at room temperature and used for protein lysis and protein determination as described in Section 4.5.

\subsection{Protein Extraction and Determination}

For measurement of total protein amount, the pellet was resuspended in $8 \mathrm{M}$ urea buffer (in $50 \mathrm{mM}$ HEPES, pH 8.5). The protein concentration was determined using a bicinchoninic acid (BCA) assay (Thermo Fischer Scientific, Waltham, MA, USA) following the manufacturer's instructions. In brief, 
$2 \mu \mathrm{L}$ from each protein lysate was added to $2 \mu \mathrm{L}$ reagent $\mathrm{A}$, followed by the addition of $100 \mu \mathrm{L}$ reagent B. After $30 \mathrm{~min}$, absorbance was read at $562 \mathrm{~nm}$ using an Infinite M200Pro plate reader (TECAN, Männedorf, Switzerland). In order to calculate a calibration curve, a dilution series of bovine serum albumin (BSA, $1 \mathrm{mg} / \mathrm{mL}$ to $0.05 \mathrm{mg} / \mathrm{mL}$ ) was included in the measurement.

\subsection{GC-MS Metabolomics Measurement of Key Central Carbon Pathway Metabolites}

All polar cell extracts were stored dry at $-80{ }^{\circ} \mathrm{C}$ until analysis. Extracts were removed from the freezer and further dried in a rotational vacuum concentrator for $60 \mathrm{~min}$ before further processing to ensure there was no residual water which may influence derivatization efficiency. Dried cell extracts were dissolved in $15 \mu \mathrm{L}$ of methoxyamine hydrochloride solution $(40 \mathrm{mg} / \mathrm{mL}$ in pyridine) and incubated for $90 \mathrm{~min}$ at $30{ }^{\circ} \mathrm{C}$ with constant shaking, followed by the addition of $50 \mu \mathrm{L}$ of $\mathrm{N}$-methyl-N-[trimethylsilyl]trifluoroacetamide (MSTFA) and incubation at $37{ }^{\circ} \mathrm{C}$ for $60 \mathrm{~min}$. The extracts were centrifuged for $10 \mathrm{~min}$ at $10,000 \times g$, and aliquots of $25 \mu \mathrm{L}$ were transferred into glass vials for GC-MS measurement. An identification mixture for reliable compound identification was prepared and derivatized in the same way and an alkane mixture for reliable retention index calculation was included [23]. Metabolite analysis was performed on a Pegasus 4D GC ×GC TOF-MS-System (LECO Corporation, St. Joseph, MN, USA) complemented with an auto-sampler (Gerstel MPS DualHead with CAS4 injector, Mühlheim an der Ruhr, Germany). The samples were injected in split mode (split 1:5, injection volume $1 \mu \mathrm{L}$ ) in a temperature-controlled injector with a baffled glass liner (Gerstel, Mühlheim an der Ruhr, Germany). The following temperature program was applied during sample injection: for $2 \mathrm{~min}$ the column was allowed to equilibrate at $68^{\circ} \mathrm{C}$, a first temperature gradient was started with a rate of increase of $5{ }^{\circ} \mathrm{C} / \mathrm{min}$ until a maximum of $120{ }^{\circ} \mathrm{C}$ was reached. Subsequently, the temperature gradient was changed such that the rate of increase was $7^{\circ} \mathrm{C} / \mathrm{min}$ up to a maximum temperature of $200{ }^{\circ} \mathrm{C}$. This was increased to a $12{ }^{\circ} \mathrm{C} / \mathrm{min}$ gradient up to a maximum temperature of $320^{\circ} \mathrm{C}$ which was then held for $7.5 \mathrm{~min}$. Gas chromatographic separation was performed on an Agilent 7890 (Agilent Technologies, Santa Clara, CA, USA), equipped with a VF-5ms column (Agilent Technologies, Santa Clara, CA, USA) of $30 \mathrm{~m}$ length, $250 \mu \mathrm{m}$ inner diameter, and $0.25 \mu \mathrm{m}$ film thickness. Helium was used as the carrier gas with a flow rate of $1.2 \mathrm{~mL} / \mathrm{min}$. The spectra were recorded in a mass range of 60 to $600 \mathrm{~m} / \mathrm{z}$ with 10 spectra/s. The GC-MS chromatograms were processed with ChromaTOF software (LECO Corporation, St. Joseph, MN, USA) including baseline assessment, peak picking, and computation of the area.

\subsection{Data Analysis}

An in-house-created library and reference search including 45 most relevant metabolites from the central carbon metabolism (CCM) were used, as seen in Table S7. The data were exported and merged by an in-house R script. The metabolites were considered valid when they appeared in a minimum of three out of five biological replicates (BR) in HCT116, two or three in female HUVECs, and two BR for male HUVECs. Male and female HUVECs were analyzed separately because of the availability of the material. The peak area of each metabolite was calculated by normalization to the internal standard, cinnamic acid, and additionally to the protein content (or cell mass). The performance of the different $\mathrm{MeOH}$ percentages were evaluated according to the following criteria: (1) number of detected metabolites, (2) relative peak area, and (3) reproducibility. The technical variation of the GC-MS run is shown in Table S8.

Supplementary Materials: The following are available online at http://www.mdpi.com/2218-1989/10/01/2/s1, Table S1. Individual metabolite relative standard deviation (RSD) of HCT116 cells cultured in $10 \%$ and $20 \%$ FCS conditions using different quenching solvents, extraction ratios, and polar volumes. *: phosphate. Table S2. Individual metabolite relative standard deviation (RSD) of HUVECs cultured in $20 \%$ FCS conditions using different quenching solvents, extraction ratios and polar volumes for the different protocols. Cells were measured in different batches (dashed line). *: 50_Medium. Table S3. Median of the relative standard deviation (RSD) per metabolite of HUVECs cultured in $20 \%$ FCS conditions using different quenching solvents, extraction ratios, and polar volumes for the different protocols. Cells were measured in different batches. Table S4. Percentage of 
metabolites with a relative standard deviation (RSD) $<30 \%$ of HUVECs cultured in $20 \%$ FCS conditions using different quenching solvents, extraction ratios, and polar volumes for the different protocols. Cells were measured in different batches. Table S5. Relative standard deviation (RSD) of measured protein amount of HUVECs cultured in $20 \%$ FCS conditions using different quenching solvents, extraction ratios, and polar volumes for the different protocols. Cells were measured in different batches. Table S6. Median of the relative standard deviation (RSD) per metabolite and percentage of metabolites with a RSD $<30 \%$ of HUVECs (three biological replicates) cultured in 20\% FCS conditions. Table S7. List of metabolite derivatives and their biological group used for reference search. AA: Amino acids. PPP: Pentose phosphate pathway. TCA: Tricarboxylic acid cycle. TMS: Trimethylsilyl derivatives. MeOX: Methoxyamine hydrochloride. Table S8. Technical variation during gas chromatography mass spectrometry (GC-MS) run of four pooled samples. RSD: Relative standard deviation. Figure S1. 50\% and 80\% of $\mathrm{MeOH}$ quenching buffer and different ratios of $\mathrm{MeOH}: \mathrm{CHCL}_{3}: \mathrm{H}_{2} \mathrm{O}$ were mixed for extraction steps. Sudan I was added to aid visualization of phase separation. No phase separation could be observed using 80_LOW condition. Therefore, the condition was not used for further testing. Figure S2. Recovery of annotated metabolites per biological group for different quenching and extraction methods. (a) HCT116 cells were cultivated in 10\% FCS (in minimum three out of five biological replicates). (b) HCT116 cells were cultivated in 20\% FCS (in minimum four out of five biological replicates). (c) HUVECs were cultured in $20 \%$ FCS (in minimum two out of three biological replicates). HCT116 cells were measured in one batch while HUVECs were measured in different batches (dashed line). The column data represents the number of annotated metabolites for each protocol. AA: Amino acids. PPP: Pentose phosphate pathway. TCA: Tricarboxylic acid cycle. Figure S3. Median relative standard deviation (RSD) of individual metabolites separated by metabolite classes from in minimum two out of three replicates of HUVECs cultured in $20 \%$ FCS conditions using different quenching solvents, extraction ratios, and polar volumes. The dashed line represents the maximum 30\% RSD threshold advised by the Food and Drug Administration (FDA). Condition details see Table 3. Cells were measured in different batches reflected by the three graphs. AA: Amino acids. PPP: Pentose phosphate pathway. TCA: Tricarboxylic acid cycle. Figure S4. Comparison of the mean of sum of normalized peak area for different quenching and extraction methods. HUVECs were cultured in $20 \%$ FCS. The cells were measured in 2 different batches (dashed line). Data from in minimum two out of three biological replicates. The peak area was normalized to cinnamic acid and protein amount. Due to the low number of biological replicates no significances were measured. File S1. Standard operating protocol for harvest and extraction of adherent cells grown under $10 \%$ or $20 \%$ fetal calf serum (FCS) conditions version 1.

Author Contributions: Conceptualization, R.F.-G. and J.A.K.; methodology, R.F.-G.; software, Y.G., R.F.-G.; formal analysis, R.F.-G.; investigation, R.F.-G.; resources, M.L., J.A.K., R.F.-G.; data curation, R.F.-G.; writing-original draft preparation, R.F.-G.; writing—review and editing, J.A.K., A.B., Y.G., M.L.; visualization, R.F.-G.; supervision, J.A.K.; project administration, J.A.K.; funding acquisition, J.A.K.

Funding: This research received no external funding.

Acknowledgments: We acknowledge Alina Eisenberger (Berlin Institute of Health Metabolomics Platform, Berlin Institute of Health (BIH), Berlin, Germany and Max-Delbrück-Center for Molecular Medicine (MDC) in the Helmholtz Association, Berlin, Germany), Cornelia Bartsch as well as Angelika Vietzke (Charité-Universitätsmedizin Berlin, corporate member of Freie Universität Berlin, Humboldt-Universität zu Berlin, and Berlin Institute of Health, Medizinische Klinik für Kardiologie und Angiologie, Campus Mitte, Berlin, Germany) for technical support. We thank the Clinic for Gynecology and Obstetrics, Charité-University Medicine Berlin for support in obtaining the umbilical cords.

Conflicts of Interest: The authors declare no conflict of interest.

\section{References}

1. Kapoore, R.V.; Vaidyanathan, S. Towards quantitative mass spectrometry-based metabolomics in microbial and mammalian systems. Philos. Trans. A Math. Phys. Eng. Sci. 2016, 374. [CrossRef] [PubMed]

2. Sellick, C.A.; Hansen, R.; Maqsood, A.R.; Dunn, W.B.; Stephens, G.M.; Goodacre, R.; Dickson, A.J. Effective quenching processes for physiologically valid metabolite profiling of suspension cultured Mammalian cells. Anal. Chem. 2009, 81, 174-183. [CrossRef] [PubMed]

3. Sellick, C.A.; Hansen, R.; Stephens, G.M.; Goodacre, R.; Dickson, A.J. Metabolite extraction from suspension-cultured mammalian cells for global metabolite profiling. Nat. Protoc. 2011, 6, 1241-1249. [CrossRef]

4. Bolten, C.J.; Kiefer, P.; Letisse, F.; Portais, J.C.; Wittmann, C. Sampling for metabolome analysis of microorganisms. Anal. Chem. 2007, 79, 3843-3849. [CrossRef]

5. Dettmer, K.; Nurnberger, N.; Kaspar, H.; Gruber, M.A.; Almstetter, M.F.; Oefner, P.J. Metabolite extraction from adherently growing mammalian cells for metabolomics studies: Optimization of harvesting and extraction protocols. Anal. Bioanal. Chem. 2011, 399, 1127-1139. [CrossRef] [PubMed]

6. Danielsson, A.P.; Moritz, T.; Mulder, H.; Spegel, P. Development and optimization of a metabolomic method for analysis of adherent cell cultures. Anal. Biochem. 2010, 404, 30-39. [CrossRef] 
7. Dietmair, S.; Timmins, N.E.; Gray, P.P.; Nielsen, L.K.; Kromer, J.O. Towards quantitative metabolomics of mammalian cells: Development of a metabolite extraction protocol. Anal. Biochem. 2010, 404, 155-164. [CrossRef]

8. Lorenz, M.A.; Burant, C.F.; Kennedy, R.T. Reducing time and increasing sensitivity in sample preparation for adherent mammalian cell metabolomics. Anal. Chem. 2011, 83, 3406-3414. [CrossRef] [PubMed]

9. Kuehnbaum, N.L.; Britz-McKibbin, P. New advances in separation science for metabolomics: Resolving chemical diversity in a post-genomic era. Chem. Rev. 2013, 113, 2437-2468. [CrossRef] [PubMed]

10. Chrysanthopoulos, P.K.; Goudar, C.T.; Klapa, M.I. Metabolomics for high-resolution monitoring of the cellular physiological state in cell culture engineering. Metab. Eng. 2010, 12, 212-222. [CrossRef] [PubMed]

11. Winder, C.L.; Dunn, W.B.; Schuler, S.; Broadhurst, D.; Jarvis, R.; Stephens, G.M.; Goodacre, R. Global metabolic profiling of Escherichia coli cultures: An evaluation of methods for quenching and extraction of intracellular metabolites. Anal. Chem. 2008, 80, 2939-2948. [CrossRef] [PubMed]

12. Sapcariu, S.C.; Kanashova, T.; Weindl, D.; Ghelfi, J.; Dittmar, G.; Hiller, K. Simultaneous extraction of proteins and metabolites from cells in culture. MethodsX 2014, 1, 74-80. [CrossRef] [PubMed]

13. Bligh, E.G.; Dyer, W.J. A rapid method of total lipid extraction and purification. Can. J. Biochem. Physiol. 1959, 37, 911-917. [CrossRef] [PubMed]

14. Folch, J.; Ascoli, I.; Lees, M.; Meath, J.A.; Le, B.N. Preparation of lipide extracts from brain tissue. J. Biol. Chem. 1951, 191, 833-841. [PubMed]

15. Folch, J.; Lees, M.; Stanley, G.H.S. A simple method for the isolation and purification of total lipides from animal tissues. J. Biol. Chem. 1957, 226, 497-509.

16. Chaipinyo, K.; Oakes, B.W.; van Damme, M.P.I. Effects of growth factors on cell proliferation and matrix synthesis of low-density, primary bovine chondrocytes cultured in collagen I gels. J. Orthop. Res. 2002, 20, 1070-1078. [CrossRef]

17. Lorenz, M.; Blaschke, B.; Benn, A.; Hammer, E.; Witt, E.; Kirwan, J.; Fritsche-Guenther, R.; Gloaguen, Y.; Bartsch, C.; Vietzke, A.; et al. Sex-specific metabolic and functional differences in human umbilical vein endothelial cells from twin pairs. Atherosclerosis 2019, 291, 99-106. [CrossRef]

18. Gupta, R.K.; Irie, R.F.; Morton, D.L. Antigens on human tumor cells assayed by complement fixation with allogeneic sera. Cancer Res. 1978, 38, 2573-2580.

19. Marin, V.; Kaplanski, G.; Gres, S.; Farnarier, C.; Bongrand, P. Endothelial cell culture: Protocol to obtain and cultivate human umbilical endothelial cells. J. Immunol. Methods 2001, 254, 183-190. [CrossRef]

20. Rosner, D.; McCarthy, N.; Bennett, M. Rapamycin inhibits human in stent restenosis vascular smooth muscle cells independently of pRB phosphorylation and p53. Cardiovasc. Res. 2005, 66, 601-610. [CrossRef]

21. Hinrichs, S.; Scherschel, K.; Kruger, S.; Neumann, J.T.; Schwarzl, M.; Yan, I.; Warnke, S.; Ojeda, F.M.; Zeller, T.; Karakas, M.; et al. Precursor proadrenomedullin influences cardiomyocyte survival and local inflammation related to myocardial infarction. Proc. Natl. Acad. Sci. USA 2018, 115, E8727-E8736. [CrossRef] [PubMed]

22. Lang, S.; Herrmann, M.; Pfeifer, C.; Brockhoff, G.; Zellner, J.; Nerlich, M.; Angele, P.; Prantl, L.; Gehmert, S.; Loibl, M. Leukocyte-reduced platelet-rich plasma stimulates the in vitro proliferation of adipose-tissue derived mesenchymal stem cells depending on PDGF signaling. Clin. Hemorheol. Microcirc. 2017, 67, 183-196. [CrossRef] [PubMed]

23. Pietzke, M.; Zasada, C.; Mudrich, S.; Kempa, S. Decoding the dynamics of cellular metabolism and the action of 3-bromopyruvate and 2-deoxyglucose using pulsed stable isotope-resolved metabolomics. Cancer Metab. 2014, 2, 9. [CrossRef] [PubMed]

24. Fritsche-Guenther, R.; Zasada, C.; Mastrobuoni, G.; Royla, N.; Rainer, R.; Rossner, F.; Pietzke, M.; Klipp, E.; Sers, C.; Kempa, S. Alterations of mTOR signaling impact metabolic stress resistance in colorectal carcinomas with BRAF and KRAS mutations. Sci. Rep. 2018, 8, 9204. [CrossRef] [PubMed]

25. Cao, B.; Aa, J.; Wang, G.; Wu, X.; Liu, L.; Li, M.; Shi, J.; Wang, X.; Zhao, C.; Zheng, T.; et al. GC-TOFMS analysis of metabolites in adherent MDCK cells and a novel strategy for identifying intracellular metabolic markers for use as cell amount indicators in data normalization. Anal. Bioanal. Chem. 2011, 400, 2983-2993. [CrossRef] [PubMed]

26. Ritter, J.B.; Genzel, Y.; Reichl, U. Simultaneous extraction of several metabolites of energy metabolism and related substances in mammalian cells: Optimization using experimental design. Anal. Biochem. 2008, 373, 349-369. [CrossRef] 
27. Dunn, W.B.; Wilson, I.D.; Nicholls, A.W.; Broadhurst, D. The importance of experimental design and QC samples in large-scale and MS-driven untargeted metabolomic studies of humans. Bioanalysis 2012, 4, 2249-2264. [CrossRef]

28. Bi, H.; Krausz, K.W.; Manna, S.K.; Li, F.; Johnson, C.H.; Gonzalez, F.J. Optimization of harvesting, extraction, and analytical protocols for UPLC-ESI-MS-based metabolomic analysis of adherent mammalian cancer cells. Anal. Bioanal. Chem. 2013, 405, 5279-5289. [CrossRef]

29. Beckonert, O.; Keun, H.C.; Ebbels, T.M.; Bundy, J.; Holmes, E.; Lindon, J.C.; Nicholson, J.K. Metabolic profiling, metabolomic and metabonomic procedures for NMR spectroscopy of urine, plasma, serum and tissue extracts. Nat. Protoc. 2007, 2, 2692-2703. [CrossRef]

30. Cuperlovic-Culf, M.; Barnett, D.A.; Culf, A.S.; Chute, I. Cell culture metabolomics: Applications and future directions. Drug Discov. Today 2010, 15, 610-621. [CrossRef]

31. Matyash, V.; Liebisch, G.; Kurzchalia, T.V.; Shevchenko, A.; Schwudke, D. Lipid extraction by methyl-tert-butyl ether for high-throughput lipidomics. J. Lipid Res. 2008, 49, 1137-1146. [CrossRef] [PubMed]

32. Muschet, C.; Moller, G.; Prehn, C.; de Angelis, M.H.; Adamski, J.; Tokarz, J. Removing the bottlenecks of cell culture metabolomics: Fast normalization procedure, correlation of metabolites to cell number, and impact of the cell harvesting method. Metabolomics 2016, 12, 151. [CrossRef] [PubMed]

33. Blankenship, D.; Niemi, J.; Hilow, E.; Karl, M.; Sundararajan, S. Oral pioglitazone reduces infarction volume and improves neurologic function following MCAO in rats. Adv. Exp. Med. Biol. 2011, 701, 157-162. [CrossRef] [PubMed]

(C) 2019 by the authors. Licensee MDPI, Basel, Switzerland. This article is an open access article distributed under the terms and conditions of the Creative Commons Attribution (CC BY) license (http://creativecommons.org/licenses/by/4.0/). 